\title{
Research Article \\ Effects of Temperature on Chronic Trapezius Myofascial Pain Syndrome during Dry Needling Therapy
}

\author{
Gang Wang, Qian Gao, Jingshan Hou, and Jun Li \\ Department of Rehabilitation Medicine, The Chinese PLA General Hospital, Beijing 100853, China \\ Correspondence should be addressed to Qian Gao; gq301@sina.com
}

Received 30 May 2014; Revised 11 August 2014; Accepted 30 August 2014; Published 14 October 2014

Academic Editor: Ching-Liang Hsieh

Copyright ( 2014 Gang Wang et al. This is an open access article distributed under the Creative Commons Attribution License, which permits unrestricted use, distribution, and reproduction in any medium, provided the original work is properly cited.

\begin{abstract}
The purpose of this study was to investigate the effects of temperature on chronic trapezius myofascial pain syndrome during dry needling therapy. Sixty patients were randomized into two groups of dry needling (DN) alone (group A) and DN combined with heat therapy group (group B). Each patient was treated once and the therapeutic effect was assessed by the visual analogue scale (VAS), pressure pain threshold (PPT), and the 36-item short form health survey (SF-36) at seven days, one month, and three months after treatment. Evaluation based on VAS and PPT showed that the pain of patients in groups A and B was significantly $(P<0.05)$ relieved at seven days, one month, and three months after treatment Compared to before treatment. There was significantly $(P<0.05)$ less pain in group B than group A at one and three months after treatment. The SF-36 evaluation demonstrated that the physical condition of patients in both groups showed significant $(P<0.05)$ improvement at one month and three months after treatment than before treatment. Our study suggests that both DN and DN heating therapy were effective in the treatment of trapezius MPS, and that DN heating therapy had better long-term effects than DN therapy.
\end{abstract}

\section{Introduction}

Myofascial pain syndrome (MPS) is a common clinical disease defined as a regional pain syndrome with characteristics of muscle pain caused by myofascial trigger points (MTrPs) [1]. MTrPs were first proposed and defined by Travell and Simons. An active MTrPs usually produces a referred pain pattern typical of that muscle, restricted range of motion and a visible or palpable local twitch response (LTR) during mechanical stimulation of the MTrPs [2]. In most cases, due to a stiff trapezius muscle, neck and upper back pain is the most common complaint in MPS [3].

Epidemiological investigations revealed that $30 \%-85 \%$ of patients with pain in the United States have MTrPs [4-6]. Among 96 patients with muscle pain who met with Gerwin [7], 74\% is caused by MTrPs. There are many treatments of MPS and dry needing (DN) therapy is increasingly used. DN is an aggressive therapy that treats lesions with dry needling alone (such as acupuncturing MTrPs), without medication. Most researchers believe that the application of DN could be traced to 1979; when Lewit published his article, the study divided the traditional drug injection treatment into two parts examining individual treatment effects, comparing the effects from drug injection and the effects from acupuncture [8]. Since then, studies on the effect of simple acupuncture have increased and are called $\mathrm{DN}$ in order to distinguish it from the traditional drug injection.

Among the patients in our outpatient clinic, those with MPS accounted for a high proportion. These patients were treated by acupuncturing MTrPs using a special kind of needle with a diameter of $0.9 \mathrm{~mm}$ that has been used for many years in our clinic. In addition, the needle tail was heated so as to increase the temperature at the needle tip based on thermal conductivity. To our knowledge, studies on effect of needle body heat in the DN treatment are not reported yet.

The purpose of this study is to investigate if the needle tail heating will improve the effect of DN in treating chronic MPS.

\section{Patients and Methods}

2.1. Patients. Patients were enrolled in Medicine Center, People's Liberation Army General Hospital, Beijing, China, from April to October 2013. The inclusion criteria included 
TABLE 1: Demographic characteristics of study participants.

\begin{tabular}{lccc}
\hline Parameter & Group A & Group B & $P$ value \\
\hline$n$ & 28 & 29 & 0.460 \\
Sex (male/female) & $11 / 17$ & $41.83 \pm 11.72$ & 0.473 \\
Age (years) & $44.07 \pm 11.71$ & $44.07 \pm 32.90$ & 0.332 \\
Disease duration (mo) & $53.04 \pm 36.30$ & $2.80 \pm 2.90$ & 0.775 \\
BMI & $23.01 \pm 2.64$ & $15 / 14$ & 0.339 \\
Location trigger point (dominant/nondominant) & $17 / 11$ & & \\
\hline
\end{tabular}

BMI: body mass index.

patients aged 20-70 years, male or female, presenting with more than three months of trapezius MPS and with moderate to severe pain with a VAS score greater than four $(0=$ no pain, 10 points $=$ worst pain). The patients had normal cognitive function, consented to participate in this study, and signed the informed consent form. The main diagnostic criteria for MPS [9] are (1) localized spontaneous pain; (2) spontaneous pain or altered sensations in expected referred pain area for given trigger point; (3) taut, palpable band in accessible muscle; (4) exquisite, localized tenderness in precise point along taut band; (5) some measurable degree of reduced range of movement. The secondary diagnostic criteria for MPS [9] are (1) reproduction of spontaneously perceived pain and altered sensations by pressure on trigger point, (2) elicitation of a local twitch response of muscular fibers by transverse "snapping" palpation or by needle insertion into trigger point, and (3) pain relief obtained by muscle stretching or injection of trigger point (Table 1).

Exclusion criteria included shoulder and neck skin disease, a history of neck surgery, use of anticoagulants, taking antiplatelet drugs within three days before the study, a history of cancer-related pain within six months before the study, receiving injections in the spot to be punctured in this study within three months before the study, receiving other treatments for MPS except for oral medications, having fibromyalgia syndrome, cervical nerve root or spinal cord lesions, a medical history of neck/shoulder operations, psychiatric or psychological disease, cancer, rheumatoid arthritis, endocrine diseases, pregnancy, severe depression or schizophrenia, obesity, and body mass index $>28$ (Table 1).

2.2. Informed Consent and Ethical Approval. Before the study, each patient meeting the inclusion criteria was provided with an informed consent form, explained about the basic procedure to be performed for treatment and evaluation and potential risk, and informed of their rights to stop treatments at any time without undertaking any consequence. Those who agreed to participate in the treatment and subsequent followup were required to sign the informed consent form for the study.

2.3. Sample Size. We refer to the previous studies to determine the sample size [10], and the sample size was initially 23 for each group here. Taking into account the possible sample loss, we determined the sample size as 30 for each group. Hence, a total of 60 patients participated in this study.
2.4. Randomly Grouping. The patients with chronic trapezius MPS enrolled in this study were randomized into two treatment groups: DN at room temperature (group A) and DN combined with heat therapy (group B). The short- and longterm treatment effects of these two methods in the patients were compared.

2.5. Single-Blind Study. Since the patients may feel the treatment difference, the single-blind study was performed following the procedure of a double-blind study in order to minimize the effects of personal emotions of patients on the treatment. Evaluations were conducted before and after treatment, and follow-up study was conducted by specialized physician who knew nothing about the treatment. Evaluation is completed by two physicians: both of them have received specialized training. After the diagnosis, the physician confirmed that patients met with inclusion criteria but without exclusion criteria and informed the patients of acupuncture therapy randomly assigned with $\mathrm{DN}$ for some and DN with heating for the others. If the patients agreed to participate in the study, the physician filled out the clinical information form, sealed, and stored it. The patients were given a card with their sequence number. On treatment, the card was collected by a triage nurse. The nurse identified the treatment group of the patients in the randomization table and told the physician. The physician could introduce the treatment procedure and precautions to patients before DN but could not mention about whether heating was applied. Data were analyzed by personnel who did not participate in the grouping and treatment.

2.6. Tool Used for the DN Treatment. The needles (Shuxin Scientific and Technology Development Co., Ltd., Shanghai, China) were made of $85 \%$ silver and smaller amounts of metals such as copper, chromium, and nickel through smelting and die-cast process. The needle handle was five $\mathrm{cm}$ in length. The needle body was eight $\mathrm{cm}$ in length and $0.9 \mathrm{~mm}$ in diameter. It was strong and flexible. The needle tip was slightly blunt.

Silver needle-conducting temperature-adjusting travelinspecting equipment, YRX-1 thermal temperature logging device (Shuxin Scientific and Technology Development Co., Ltd., Shanghai, China), was used as the heating instrument. It had 20 bamboo-style heating tubes placed around the needle handle for resistance wire heating. It was attached with temperature feedback loop with the highest temperature of 


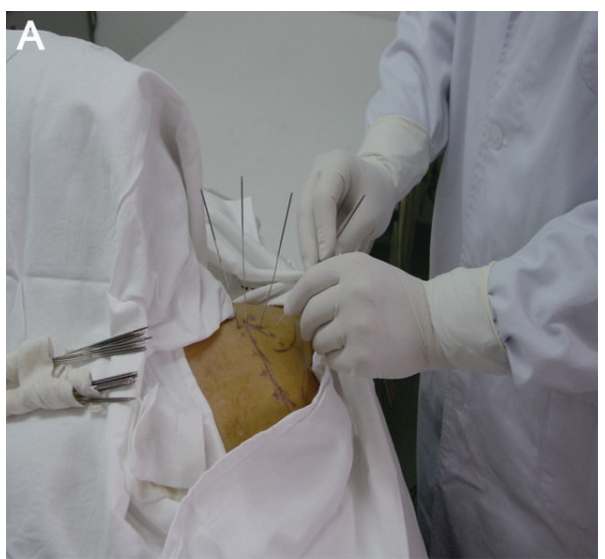

(a) Dry needle therapy

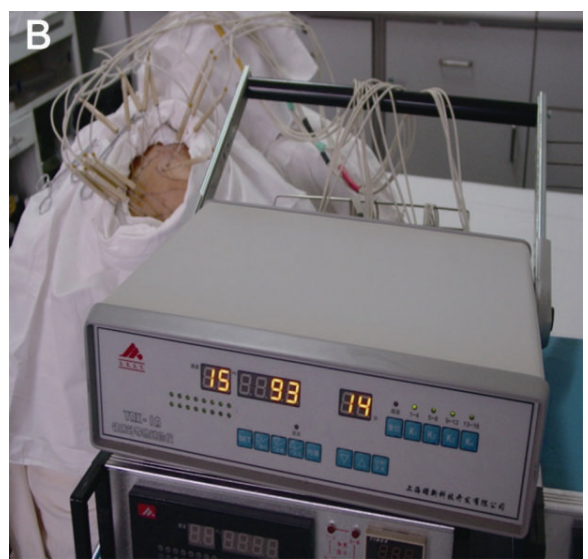

(b) Dry needle heating special instrument

FIGURE 1: Myofascial pain syndrome treated with dry needle therapy.

$120^{\circ} \mathrm{C}$ and temperature fluctuation less than $\pm 2^{\circ} \mathrm{C}$ around the target temperature.

Digital thermometer (Nanjing University of Science \& Technology, Nanjing, China) was used. It was four-channel with a measurement range of $15-99^{\circ} \mathrm{C}$, accuracy of $0.5^{\circ} \mathrm{C}$, and the reaction time of $0.85 \mathrm{~s}$. It included the needle semiconductor thermistor sensor with temperature needle length of $12 \mathrm{~cm}$ and diameter of $1 \mathrm{~mm}$.

Pressure pain threshold analyzer was the SLY-HFM hand type pain threshold detector (Beijing Shuolin Scientific and Technology Development Co., Ltd., Beijing, China) with a measurement range of $0-1000 \mathrm{~g}$, an accuracy of $2.5 \mathrm{~g}$, and a precision of $0.1 \mathrm{~g}$.

2.7. Procedure. Patients in group A were treated with $\mathrm{DN}$ in a diameter of $0.9 \mathrm{~mm}$ while those in group B were treated with DN plus additional 15 minutes of heat treatment. Specifically, patients in group A lied in bed in a prone position. The spot of trapezius MTrP was determined and marked by an experienced and licensed physician. After sterilization with disinfectant and draping were completed, the doctor wore sterile gloves to identify the MTrP using the left hand and intradermally injected $0.25 \%$ lidocaine at each point. When a local hillock in diameter of about $0.8 \mathrm{~mm}$ was formed, the needle in the right hand was injected vertically into the skin to reach the MTrP (the correct position was assumed as long as the usual symptoms of pain or local twitch response were observed [11]). The treatment above was performed on each MTrP for 15 min. Patients in group B experienced the acupuncture following the procedure same as above, but the needles were heated using a special heating device by covering bamboo-style heating tubes over each needle tail. The thermodetector was applied to measure and adjust the temperature so as to ensure the injection temperature at $44^{\circ} \mathrm{C}$. After $15 \mathrm{~min}$ of acupuncture, the needle was removed. Because the type of needle used was thick, a small amount of blood may ooze upon withdrawal. In order to reduce bleeding and to prevent infection, sterile gauze was used for local oppression for three min after needle removal. Then, sterile gauze was replaced to cover the treated area and fixed with

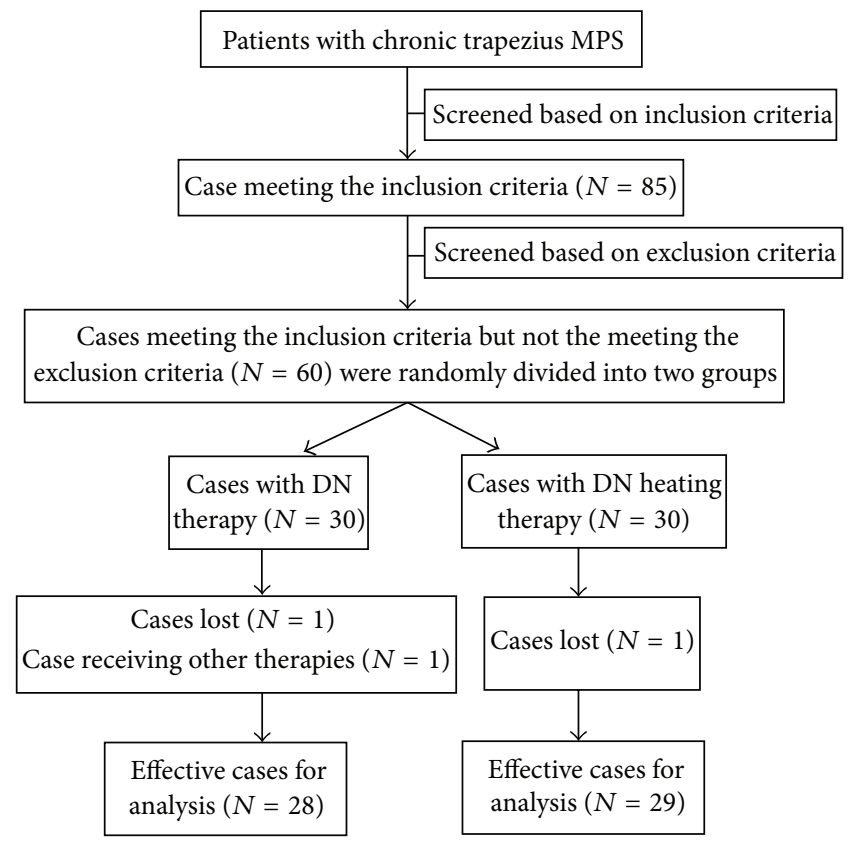

FIGURE 2: A heating instrument for dry needling therapy.

adhesive tape. The patients were told to avoid contact with water in the treatment spots for 48 hours. After that, the tape could be removed. No further medication or physical therapy was performed (Figures 1 and 2).

2.8. Evaluation Method. The effects of DN on MPS were evaluated according to the visual analog scale (VAS) and shortform 36-item questionnaire (SF-36) [10, 12]. The pressure pain threshold (PPT) was also considered because the VAS and SF-36 methods could be affected by subjective factors.

The PPT was determined by a handheld PPT detector as followed. The detector is composed of a pressure rod about one $\mathrm{cm}^{2}$ attached with the rubber pad at one end, which was connected with a circular pressure gauge at the other end through the connecting rod with built-in piston. 
TABLE 2: Visual analogue scale (mean $\pm \mathrm{SD})$.

\begin{tabular}{|c|c|c|c|c|c|c|}
\hline \multicolumn{7}{|c|}{ VAS } \\
\hline Parameter & $0 \mathrm{~d}$ & $7 \mathrm{~d}$ & $1 \mathrm{~m}$ & $3 \mathrm{~m}$ & \multicolumn{2}{|c|}{$\mathrm{p}$} \\
\hline \multirow{3}{*}{ A group } & & & & & $\mathrm{p} 1=0.014$ & $\mathrm{p} 2=0.005$ \\
\hline & $6.5 \pm 1.2$ & $5.7 \pm 1.3$ & $5.5 \pm 1.3$ & $5.8 \pm 1.2$ & p3 $=0.034$ & $\mathrm{p} 4=0.708$ \\
\hline & & & & & p5 $=0.723$ & $\mathrm{p} 6=0.466$ \\
\hline \multirow{3}{*}{ B group } & & & & & $\mathrm{p} 1=0.003$ & $\mathrm{p} 2=0.000$ \\
\hline & $6.4 \pm 1.1$ & $5.1 \pm 1.7$ & $4.6 \pm 1.9$ & $4.7 \pm 2.1$ & $\mathrm{p} 3=0.000$ & $\mathrm{p} 4=0.342$ \\
\hline & & & & & $\mathrm{p} 5=0.454$ & $\mathrm{p} 6=0.840$ \\
\hline$P$ & 0.827 & 0.15 & 0.043 & 0.024 & & \\
\hline
\end{tabular}

p1: comparison between 0 and seven days of treatment; $\mathrm{p} 2$ : comparison between 0 and one month of treatment; p3: comparison between 0 and three months of treatment; p4: comparison between seven days and one month of treatment; p5: comparison between seven days and one month of treatment; p6: comparison between one month and three months of treatment.

The gauge had the subgrids with $100 \mathrm{~g}$ for each subgrid in units of $\mathrm{kg} / \mathrm{cm}^{2}$, a measurement range of $1-10 \mathrm{~kg} / \mathrm{cm}^{2}$. For use, the end with the rubber pad was directed at the measurement point. The long axis of PPT was perpendicular to the skin surface over the measurement point. Then, the pressure was increased with a rate of $30 \mathrm{kPa} / \mathrm{s}$ [13]. The pressurization stopped when the patients started to feel and complained about the pain. The pressure bar was removed way from the measurement point. At this moment, the value displayed on the gauge was PPT of the measurement point. When the measurement was finished, the gauge was set to zero by pressing the zero button and pointing the pointer to its initial position, making it ready for the next measurement. The point for treatment and used as control was individually measured. During the measurement, each point was detected for three times with a measurement interval of $15 \mathrm{~s}$. The average of three measurements was recorded for each point.

2.9. Statistical Analysis. Data were analyzed using the Statistical Package for Social Science version 13 (SPSS 13). The Chi-square test was performed for comparison of gender, age, disease duration, and whether the trigger points were located in the dominant side in patients among the two groups. Analysis of variance was used to compare the difference before and after treatments in each group. The $t$-test was for intergroup comparison at the same treatment time. A probability smaller than $0.05(P<0.05)$ was considered to be statistically significant. The treatment effects in patients were evaluated at 0 days (before treatment), seven days (after treatment), one month (after treatment), and three months (after treatment).

\section{Results}

VAS analogue scale showed that the pain in patients of group A was relieved after seven days, one month, and three months after treatment than before treatment. But there were not significantly different of pain in seven days, one month, and three months after treatment. Similarly, the pain in patients of group B was also relieved after seven days, one month, and three months after treatment than before treatment. And there were not significantly different of pain in seven days, one month, and three months after treatment (Table 2).

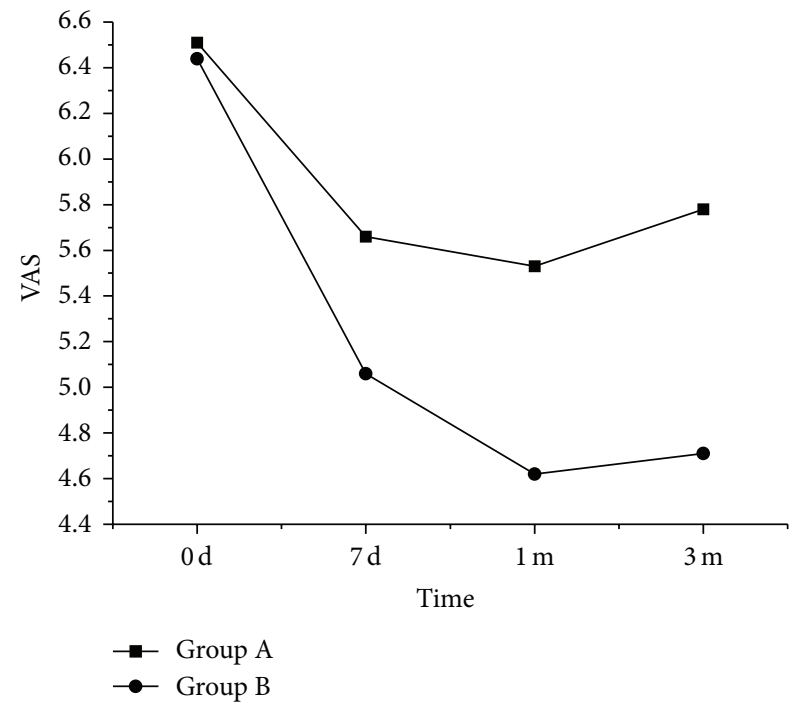

FIGURE 3: VAS analogue scales before and after treatment in patients of groups $\mathrm{A}$ and $\mathrm{B}$.

The degree of pain in patients was similar between groups $\mathrm{A}$ and $\mathrm{B}$ before treatment and in seven days of treatment. However, the pain in patients of group B was lighter than that in group A in one and three months of treatment (Figure 3).

The SF-36-based evaluation demonstrated that, compared with before treatment, the quality of life of patients in group A was not improved after seven days of treatment but greatly improved in one and three months after treatment. There was no significant difference in quality of life of patients of group A between seven days of treatment and one month of treatment and between one and three months of treatment. However, the quality of life of patients in group A was better in three months of treatment than in seven days of treatment. Compared with before treatment, the quality of life of patients in group B was not improved after seven days of treatment but greatly improved in one and three months after treatment. There was no significant difference in quality of life of patients of group B between one and three months of treatment. However, the quality of life of patients in group B was better in one and three months of treatment than in seven days of treatment (Table 3). 
TABLE 3: SF-36 evaluation before and after treatment in patients of groups A and B (mean \pm SD).

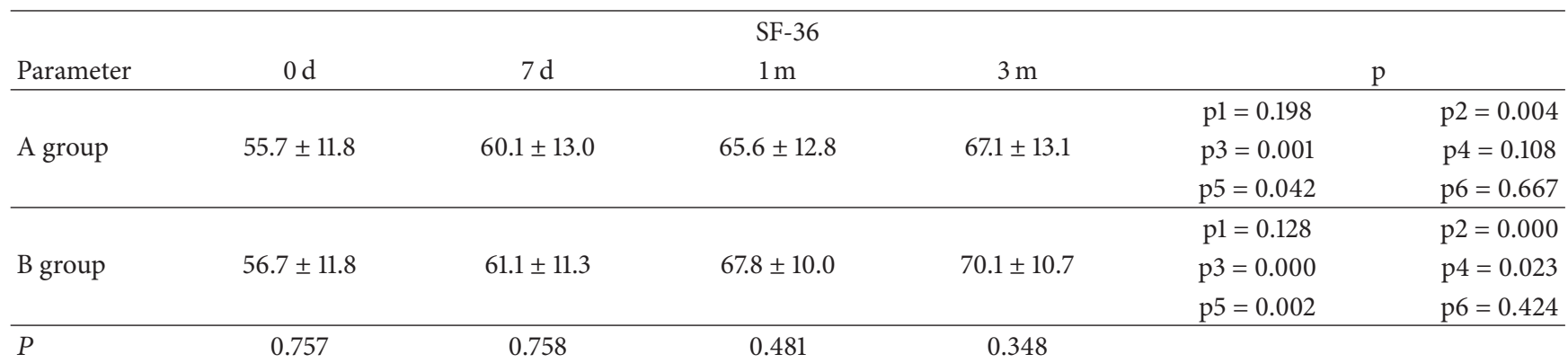

p1: comparison between 0 and seven days of treatment; p2: comparison between 0 and one month of treatment; $\mathrm{p} 3$ : comparison between 0 and three months of treatment; p4: comparison between seven days and one month of treatment; p5: comparison between seven days and one month of treatment; p6: comparison between one month and three months of treatment.

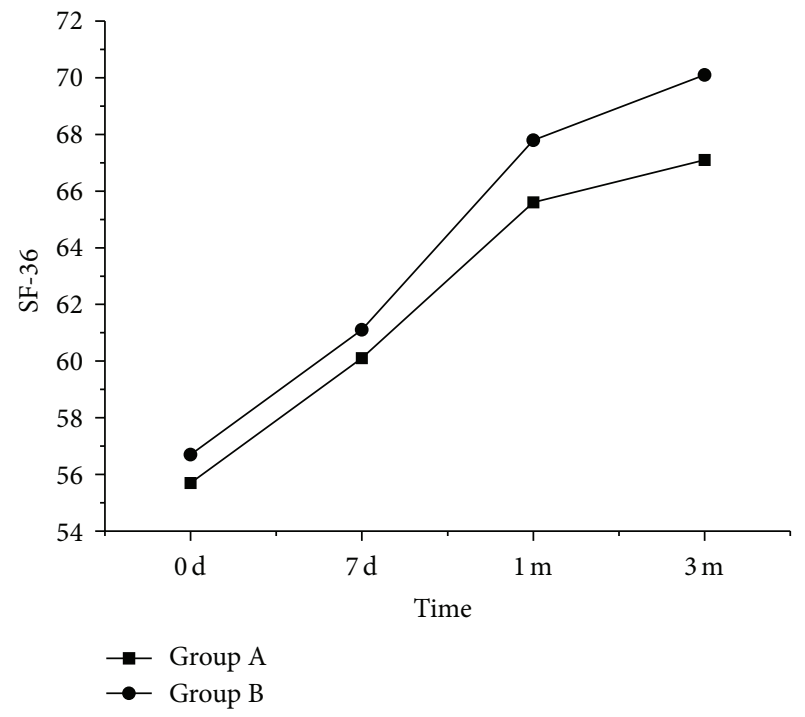

FIGURE 4: SF-36 scale changes before and after treatment in patients of groups $\mathrm{A}$ and $\mathrm{B}$.

The quality of life of patients was similar between groups $\mathrm{A}$ and $\mathrm{B}$ before treatment, in seven days of treatment, and in one and three months of treatment (Figure 4).

The results above based on SF-36 evaluation suggested that the quality of life of patients with chronic trapezius MPS could be improved in one to three months after treatment using simply DN and DN heating therapy. The effects of the two treatments on the quality of life of patients MPS were similar in seven days, one month, and three months after treatment.

The PPT values showed that the pain in patients of group A was relieved after seven days, one month, and three months after treatment than before treatment. But the PPT values were similar in seven days, one month, and three months after treatment. The pain in patients of group B was relieved after seven days, one month, and three months after treatment more than before treatment, and after one month, and three months than after seven days, but not significantly different in one and three months after treatment (Table 4).

The degree of pain in patients was similar between groups $\mathrm{A}$ and $\mathrm{B}$ before treatment and in seven days of treatment.

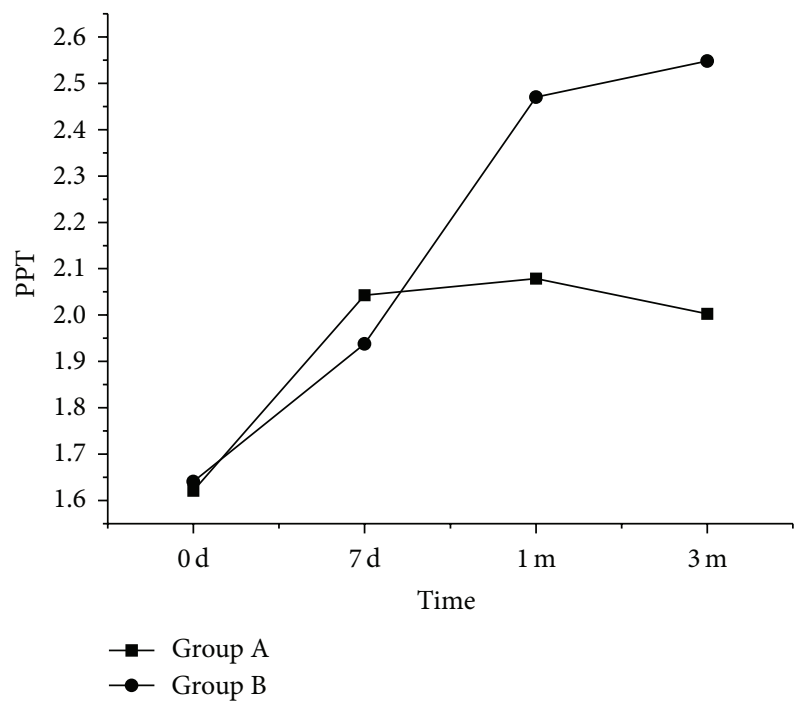

FIGURE 5: PPT values in patients of group A and group B before and after treatment.

However, the pain in patients of group B was lighter than that in group $\mathrm{A}$ in one and three months of treatment (Figure 5).

\section{Discussion}

Hot pack therapy can alleviate pain in patients with MPS [14] and improve the range of motion [15]. Basic study has demonstrated that the pathophysiological basis of MPS includes the formation of taut band and local hypoxia and ischemia [16], while heating can increase local blood flow and oxygen supply to the local tissues, local blood flow can quickly be restored to normal condition after heat source is removed, and the increase in oxygen supply can last for a long time [17]. Hot pack therapy is an effective treatment for MPS, but its effects are not as good as the combination therapy [18], and thus the MPS is rarely treated with hot pack therapy alone clinically.

$\mathrm{DN}$ is one of the methods used for treating MPS. Vulfsons et al. [19] considered that DN is effective in the treatment of myofascial trigger point. Kietrys et al. [20], through a systematic review and meta-analysis, suggested that $\mathrm{DN}$ is 
TABLE 4: Pressure pain threshold (mean \pm SD).

\begin{tabular}{|c|c|c|c|c|c|c|}
\hline \multicolumn{7}{|c|}{$\mathrm{PPT} \mathrm{kg} / \mathrm{cm}^{2}$} \\
\hline Parameter & $0 \mathrm{~d}$ & $7 \mathrm{~d}$ & $1 \mathrm{~m}$ & $3 \mathrm{~m}$ & \multicolumn{2}{|c|}{$\mathrm{p}$} \\
\hline \multirow{3}{*}{ A group } & \multirow{3}{*}{$1.62 \pm 0.51$} & \multirow{3}{*}{$2.04 \pm 0.75$} & \multirow{3}{*}{$2.07 \pm 0.76$} & \multirow{3}{*}{$2.00 \pm 0.70$} & $\mathrm{p} 1=0.024$ & $\mathrm{p} 2=0.015$ \\
\hline & & & & & $\mathrm{p} 3=0.040$ & $\mathrm{p} 4=0.847$ \\
\hline & & & & & $\mathrm{p} 5=0.832$ & $\mathrm{p} 6=0.685$ \\
\hline \multirow{3}{*}{ B group } & \multirow{3}{*}{$1.64 \pm 0.31$} & \multirow{3}{*}{$1.94 \pm 0.48$} & \multirow{3}{*}{$2.47 \pm 0.63$} & \multirow{3}{*}{$2.55 \pm 0.68$} & $\mathrm{p} 1=0.018$ & $\mathrm{p} 2=0.000$ \\
\hline & & & & & $\mathrm{p} 3=0.000$ & $\mathrm{p} 4=0.016$ \\
\hline & & & & & p5 $=0.012$ & $\mathrm{p} 6=0.911$ \\
\hline$P$ & 0.859 & 0.532 & 0.039 & 0.004 & & \\
\hline
\end{tabular}

p1: comparison between 0 and 7 days of treatment; p2: comparison between 0 and 1 month of treatment; p3: comparison between 0 and 3 months of treatment; p4: comparison between 7 days and 1 month of treatment; p5: comparison between 7 days and 1 month of treatment; p6: comparison between 1 month and 3 months of treatment.

more effective in the treatment of MPS in upper extremities than conventional physical therapy, and its evidence-based medicine level achieves grade A.

In this study, the clinical observations confirmed that the effect of DN heating therapy on MPS was better than DN therapy alone.

The DN heating therapy is innovative. Traditional Chinese acupuncture can also be performed under the heating condition usually by ignited moxa stick attached at the needle tail, which is effective in the treatment of MPS [21] but different from the treatment here. The heat from ignited moxa stick at the needle tail can mainly radiate the superficial parts of the skin and subcutaneous tissue but is difficult to reach deep tissue through heat transfer. It was because the stainless steel needles used for the clinical treatment of MPS are usually thin in diameter of $0.25-0.5 \mathrm{~mm}$ [22-25] and the thermal conductivity of stainless steel is relatively low with about $80 \mathrm{~W} / \mathrm{mK}$ so that the heat transferred from the tail into the human body is quickly taken away at the superficial tissue and the temperature at the needle tip does not change significantly before and after heating [26]. In this study, the needle was made of a variety of metals including $85 \%$ silver with thermal conductivity of $429 \mathrm{~W} / \mathrm{mK}$ and smaller amounts of other metals such as copper, chromium, and nickel, with thermal conductivity higher than stainless steel. In addition, the needle diameter was $0.9 \mathrm{~mm}$ and could quickly transfer heat from the needle tail to the needle tip. In spite of a large amount of heat absorbed by superficial tissue, some heat was still transmitted to the needle tip. Previous studies have demonstrated that heating the needle tail can increase temperature at the tip of the needle mainly made of silver $[27,28]$. Therefore, the DN heating therapy studied could heat deep tissues, especially taut bands that the needle tip could reach, thereby improving the ischemic-hypoxic condition of lesions and clinical efficacy.

The heating device used in this study could achieve stable heating and precise temperature adjustment. After reaching the target value in one-two min, the temperature detected by temperature-measuring device through feedback of bamboostyle heating apparatus was kept constant, which met the requirements for treatment and ensured that patients were not damaged by high temperature.

\subsection{Changes in Pain Intensity}

4.1.1. VAS and PPT. The VAS method can be affected by many subjective factors such as mental stress [29], but it is simple and easy to operate and understand by patients; thus it has good reliability and validity [30] and is still widely used in clinical practice.

This study based on the VAS evaluation showed that both DN only and DN heating therapy were effective for chronic and trapezius MPS, and the therapeutic effect could last for at least three months. After one and three months of treatment, DN heating treatment had better effects on MPS than on the DN treatment.

The quantitative measurement of PPT was first proposed by Libman [31] in 1934. Hereafter, PPT determination has been increasingly used in clinical treatment. Studies have confirmed high reliability of PPT measurement in clinical treatment $[32,33]$ and for the healthy population [34]. The PPT has been widely applied for evaluation of MPS and various skeletal muscle diseases [35] and evidenced for its good reliability and validity in the evaluation of MPS [36].

Current researches on PPT mostly focus on the trapezius muscle and infraspinatus. It was reported that the PPT of trapezius muscle is $3.7 \mathrm{~kg} / \mathrm{cm}^{2}$ in normal female adult [35], $2.3 \mathrm{~kg} / \mathrm{cm}^{2}$ in female telephone operator [37], and $3.1 \mathrm{~kg} / \mathrm{cm}^{2}$ in normal male adult [38]. Kwon et al. [39] detected 20 female patients with MPS and found the PPF averaged $5.4 \mathrm{~kg} / \mathrm{cm}^{2}$. This study revealed that the PPT in patients with MPS was $1.6 \mathrm{~kg} / \mathrm{cm}^{2}$ before treatment, $2.0 \mathrm{~kg} / \mathrm{cm}^{2}$ after DN treatment, and $2.0 \mathrm{~kg} / \mathrm{cm}^{2}$ after DN heating treatment. The results were consistent with those found by Lee et al. [37] but different from those reported by Lee et al. [38], which may be caused by factors such as operating skills, measurement tools, gender of patients, dominant location of trigger points, and body mass index [36].

This study based on PPT method demonstrated that both DN and DN heating treatment could treat MPS and the therapeutic effect could last at least for three months. In one and three months of treatment, the effect of DN heating treatment was better than that of DN treatment.

4.1.2. Quality of Life. SF-36 is the most widely used tool for physical and mental health assessment and is increasingly 
applied to evaluate patients with pain [40, 41]. Its evaluation is based on the answers of patients to eight healthrelated questions and one question about changes in their physical condition in the past year [42]. Its reliability and validity for evaluation of chronic pain disorders have been confirmed [29, 43]. The eight health-related questions are (1) limitations on physical activities because of health problems, (2) limitations on social activities because of physical or emotional problems, (3) limitations on usual role activities because of physical health problems, (4) bodily pain, (5) general mental health (psychological distress and wellbeing), (6) limitations on usual role activities because of emotional problems, (7) vitality (energy and fatigue), and (8) general health perceptions [44]. The Chinese version of the SF-36 rating scale was used here. SF-36 score ranged from 0 to 100 , with 0 suggesting poor physical condition and 100 indicating the best physical condition.

The SF-36-based evaluation here showed that both methods were effective for chronic trapezius MPS, and their effects were not different, which was different from the results derived from VAS and PPT evaluation. It was probably because the DN treatment including TN heating therapy primarily focused on the pain in patients. The VAS and PPT were used to assess the degree of pain and more sensitive to the degree of pain, while the SF-36 is mainly for evaluation of the overall physical condition of patients that included pain and others. Although pain might be included in the other items, it did not account for a high proportion in the SF-36 evaluation and hence SF-36 evaluation was less sensitive to pain changes than VAS and PPT.

The limitations of the DN heating treatment were associated with the large diameter of needles. Although lidocaine was used for surface anesthesia before acupuncture, patients still could feel the obvious pain during the treatment because the needle was injected into a relatively deep position. Fortunately, the pain was within the acceptable range of most patients, occurred mainly in the process that the needle was inserted, and was not obvious when the needle remained in the body and heated, which was evidenced by no sample loss during the treatment due to pain caused by inserting needle.

\section{Conclusions}

Dry needle treatment of chronic trapezius muscle myofascial pain syndrome is effective. And our research showed that doing needle treatment conduction through the needle tail heating was safe, evaluation methods of VAS and PPT confirmed that heating dry needle in improving the degree of pain had a better long-term curative effect than just dry needle therapy.

\section{Conflict of Interests}

The authors declare that there is no conflict of interests regarding the publication of this paper.

\section{Acknowledgment}

The authors are grateful to Zhili Yang, Fei Wang, and Wenyu Zhang for assistance in the whole experiment. This study was supported by the National Natural Science Foundation Fund of China for study on quantitative evaluation system of myofascial tension band. This study was also supported by Grant from the Wu JiePing Medical Foundation (320.6750.1234).

\section{References}

[1] S. A. Kim, K. Y. Oh, W. H. Choi, and I. K. Kim, "Ischemic compression after trigger point injection affect the treatment of myofascial trigger points," Annals of Rehabilitation Medicine, vol. 37, no. 4, pp. 541-546, 2013.

[2] C.-Z. Hong, "Lidocaine injection versus dry needling to myofascial trigger point: the importance of the local twitch response," American Journal of Physical Medicine and Rehabilitation, vol. 73, no. 4, pp. 256-263, 1994.

[3] Ü. Dündar, Ö. Solak, F. Şamli, and V. Kavuncu, "Effectiveness of ultrasound therapy in cervical myofascial pain syndrome: a double blind, placebo-controlled study," Turkish Journal of Rheumatology, vol. 25, no. 3, pp. 110-115, 2010.

[4] D. A. Fishbain, M. Goldberg, B. Robert Meagher, R. Steele, and H. Rosomoff, "Male and female chronic pain patients categorized by DSM-III psychiatric diagnostic criteria," Pain, vol. 26, no. 2, pp. 181-197, 1986.

[5] J. R. Fricton, R. Kroening, D. Haley, and R. Siegert, "Myofascial pain syndrome of the head and neck: a review of clinical characteristics of 164 patients," Oral Surgery Oral Medicine and Oral Pathology, vol. 60, no. 6, pp. 615-623, 1985.

[6] S. A. Skootsky, B. Jaeger, and R. K. Oye, "Prevalence of myofascial pain in general internal medicine practice," Western Journal of Medicine, vol. 151, no. 2, pp. 157-160, 1989.

[7] R. Gerwin, "A study of 96 subjects examined both for fibromyalgia and myofascial pain," Journal of Musculoskeletal Pain, vol. 3, supplement 1, p. 121, 1995.

[8] K. Lewit, "The needle effect in the relief of myofascial pain," Pain, vol. 6, no. 1, pp. 83-90, 1979.

[9] S. Ay, Ş. K. Doğan, D. Evcik, and Ö. Ç. Başer, "Comparison the efficacy of phonophoresis and ultrasound therapy in myofascial pain syndrome," Rheumatology International, vol. 31, no. 9, pp. 1203-1208, 2011.

[10] L. Tekin, S. Akarsu, O. Durmuş, E. Çakar, Ü. Dinçer, and M. Z. Kiralp, "The effect of dry needling in the treatment of myofascial pain syndrome: a randomized double-blinded placebocontrolled trial," Clinical Rheumatology, vol. 32, no. 3, pp. 309315, 2013.

[11] M. Abbaszadeh-Amirdehi, N. N. Ansari, S. Naghdi, G. Olyaei, and M. R. Nourbakhsh, "The neurophysiological effects of dry needling in patients with upper trapezius myofascial trigger points: study protocol of a controlled clinical trial," BMJ Open, vol. 3, no. 5, Article ID e002825, 2013.

[12] S.-H. Yoon, U. W. Rah, S. S. Sheen, and K. H. Cho, "Comparison of 3 needle sizes for trigger point injection in myofascial pain syndrome of upper- and middle-trapezius muscle: a randomized controlled trial," Archives of Physical Medicine and Rehabilitation, vol. 90, no. 8, pp. 1332-1339, 2009.

[13] B. Börsbo, G. M. Liedberg, M. Wallin, and B. Gerdle, "Subgroups based on thermal and pressure pain thresholds in women with chronic whiplash display differences in clinical 
presentation-an explorative study," Journal of Pain Research, vol. 5, pp. 511-521, 2012.

[14] C.-R. Hou, L.-C. Tsai, K.-F. Cheng, K.-C. Chung, and C.Z. Hong, "Immediate effects of various physical therapeutic modalities on cervical myofascial pain and trigger-point sensitivity," Archives of Physical Medicine and Rehabilitation, vol. 83, no. 10, pp. 1406-1414, 2002.

[15] J. Kain, L. Martorello, E. Swanson, and S. Sego, "Comparison of an indirect tri-planar myofascial release (MFR) technique and a hot pack for increasing range of motion," Journal of Bodywork and Movement Therapies, vol. 15, no. 1, pp. 63-67, 2011.

[16] B. Cagnie, V. Dewitte, T. Barbe, F. Timmermans, N. Delrue, and M. Meeus, "Physiologic effects of dry needling," Current Pain and Headache Reports, vol. 17, no. 8, p. 348, 2013.

[17] K. Kubo, H. Yajima, M. Takayama, T. Ikebukuro, H. Mizoguchi, and N. Takakura, "Effects of acupuncture and heating on blood volume and oxygen saturation of human Achilles tendon in vivo," European Journal of Applied Physiology, vol. 109, no. 3, pp. 545-550, 2010.

[18] B. Acar and Ö. T. Yilmaz, "Effects of different physiotherapy applications on pain and mobility of connective tissue in patients with myofascial pain syndrome," Journal of Back and Musculoskeletal Rehabilitation, vol. 25, no. 4, pp. 261-267, 2012.

[19] S. Vulfsons, M. Ratmansky, and L. Kalichman, "Trigger point needling: techniques and outcome," Current Pain and Headache Reports, vol. 16, no. 5, pp. 407-412, 2012.

[20] D. M. Kietrys, K. M. Palombaro, E. Azzaretto et al., "Effectiveness of dry needling for upper-quarter myofascial pain: a systematic review and meta-analysis," Journal of Orthopaedic \& Sports Physical Therapy, vol. 43, no. 9, pp. 620-634, 2013.

[21] R.-X. Chen, M.-F. Kang, W.-L. He, S.-Y. Chen, and B. Zhang, "Moxibustion on heat-sensitive acupoints for treatment of myofascial pain syndrome: a multi-central randomized controlled trial," Chinese Acupuncture \& Moxibustion, vol. 28, no. 6, pp. 395-398, 2008.

[22] Y.-T. Huang, S.-Y. Lin, C.-A. Neoh, K.-Y. Wang, Y.-H. Jean, and H.-Y. Shi, "Dry needling for myofascial pain: prognostic factors," The Journal of Alternative and Complementary Medicine, vol. 17, no. 8, pp. 755-762, 2011.

[23] Y.-L. Hsieh, L.-W. Chou, Y.-S. Joe, and C.-Z. Hong, "Spinal cord mechanism involving the remote effects of dry needling on the irritability of myofascial trigger spots in rabbit skeletal muscle," Archives of Physical Medicine and Rehabilitation, vol. 92, no. 7, pp. 1098-1105, 2011.

[24] Y. L. Hsieh, M. J. Kao, T. S. Kuan, S. M. Chen, J.-T. Chen, and C.Z. Hong, "Dry needling to a key myofascial trigger point may reduce the irritability of satellite MTrPs," The American Journal of Physical Medicine and Rehabilitation, vol. 86, no. 5, pp. 397403, 2007.

[25] C.-T. Tsai, L.-F. Hsieh, T.-S. Kuan, M.-J. Kao, L.-W. Chou, and C.-Z. Hong, "Remote effects of dry needling on the irritability of the myofascial trigger point in the upper trapezius muscle," American Journal of Physical Medicine and Rehabilitation, vol. 89, no. 2, pp. 133-140, 2010.

[26] L. Guan, X. Shi, Y. Zou, X.-F. Deng, and P.-S. Cai, "Effect on body surface thermograph in patients with myofascial pain syndrome treated with moxibustion on Yanglingquan (GB 34)," Chinese Acupuncture \& Moxibustion, vol. 30, no. 6, pp. 485-489, 2010.
[27] W. Fei, G. Qian, Y. Zhi-li, and W. Gang, "Study on temperature change of silver needle in vitro," Clinical Journal of Medical, vol. 38, no. 1, pp. 19-22, 2010.

[28] F. Wang, Q. Gao, G. Wang, and Z. Yang, "Change of temperature of silver needle inside isolated skeletal muscles of pig," Chinese Journal of Health Care and Medicine, vol. 5, p. 12, 2009.

[29] F.-X. Lesage, S. Berjot, and F. Deschamps, "Clinical stress assessment using a visual analogue scale," Occupational Medicine, vol. 62, no. 8, pp. 600-605, 2012.

[30] B. S. Sindhu, O. Shechtman, and L. Tuckey, "Validity, reliability, and responsiveness of a digital version of the visual analog scale," Journal of Hand Therapy, vol. 24, no. 4, pp. 356-364, 2011.

[31] E. Libman, "Observations on individual sensitiveness to pain: with special reference to abdominal disorders," Journal of the American Medical Association, vol. 102, no. 5, pp. 335-341, 1934.

[32] D. Maquet, J.-L. Croisier, C. Demoulin, and J.-M. Crielaard, "Pressure pain thresholds of tender point sites in patients with fibromyalgia and in healthy controls," European Journal of Pain, vol. 8, no. 2, pp. 111-117, 2004.

[33] N. Smidt, D. A. Van der Windt, W. J. Assendelft et al., "Interobserver reproducibility of the assessment of severity of complaints, grip strength, and pressure pain threshold in patients with lateral epicondylitis," Archives of Physical Medicine and Rehabilitation, vol. 83, no. 8, pp. 1145-1150, 2002.

[34] A. L. Persson, C. Brogådh, and B. H. Sjölund, “Tender or not tender: test-retest repeatability of pressure pain thresholds in the trapezius and deltoid muscle of healthy women," Journal of Rehabilitation Medicine, vol. 36, no. 1, pp. 17-27, 2004.

[35] A. A. Fischer, "Pressure threshold meter: its use for quantification of tender spots," Archives of Physical Medicine and Rehabilitation, vol. 67, no. 11, pp. 836-838, 1986.

[36] G. Park, C. W. Kim, S. B. Park, M. J. Kim, and S. H. Jang, "Reliability and usefulness of the pressure pain threshold measurement in patients with myofascial pain," Annals of Rehabilitation Medicine, vol. 35, no. 3, pp. 412-417, 2011.

[37] G. H. Lee, S. B. Park, S. G. Lee, K. M. Lee, S. C. Roh, and J. Song, "Pressure threshold, grip and pinch strength in female telephone operators," Journal of Korean Academy of Rehabilitation Medicine, vol. 21, no. 3, pp. 589-593, 1997.

[38] J. H. Lee, H. S. Shin, C. H. Yoon, M. K. Oh, and S. H. Kwon, "Usefulness of electronic pressure algometer in evaluation of pressure pain threshold in normal Korean adults," Journal of Korean Academy of Rehabilitation Medicine, vol. 32, no. 6, pp. 698-702, 2008.

[39] Y. E. Kwon, S. J. Lee, C. S. Yoon, and J. H. Lee, "Pressure pain threshold measurement using a pressure algometer in myofascial pain syndromes," Journal of the Korean Pain Society, vol. 14, no. 1, pp. 32-36, 2001.

[40] P. Bech, "Health-related quality of life measurements in the assessment of pain clinic results," Acta Anaesthesiologica Scandinavica, vol. 43, no. 9, pp. 893-896, 1999.

[41] B. H. Smith, K. I. Penny, A. M. Purves et al., "The chronic pain grade questionnaire: validation and reliability in postal research," Pain, vol. 71, no. 2, pp. 141-147, 1997.

[42] S. S. Farivar, W. E. Cunningham, and R. D. Hays, "Correlated physical and mental health summary scores for the SF-36 and SF-12 Health Survey, V.1," Health and Quality of Life Outcomes, vol. 5, article 54, 2007. 
[43] E. A. Schlenk, J. A. Erlen, J. Dunbar-Jacob et al., "Health-related quality of life in chronic disorders: a comparison across studies using the MOS SF-36," Quality of Life Research, vol. 7, no. 1, pp. 57-65, 1998.

[44] M. F. M. Aranha, M. C. Alves, F. Bérzin, and M. B. D. Gavião, "Efficacy of electroacupuncture for myofascial pain in the upper trapezius muscle: a case series," Revista Brasileira de Fisioterapia, vol. 15, no. 5, pp. 371-379, 2011. 


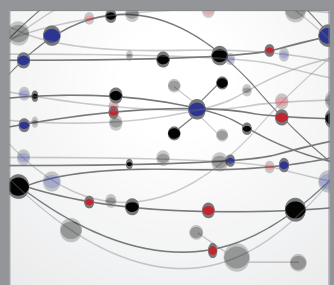

The Scientific World Journal
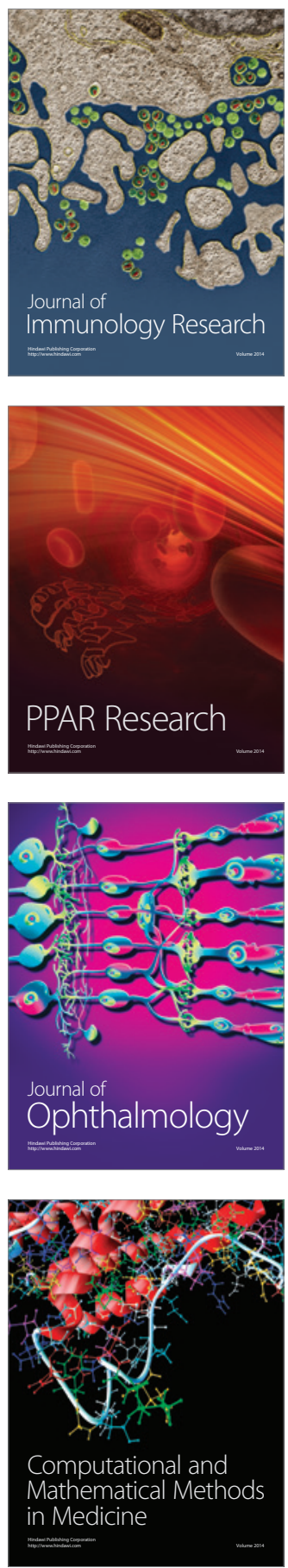

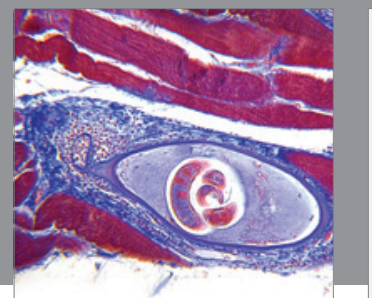

Gastroenterology

Research and Practice
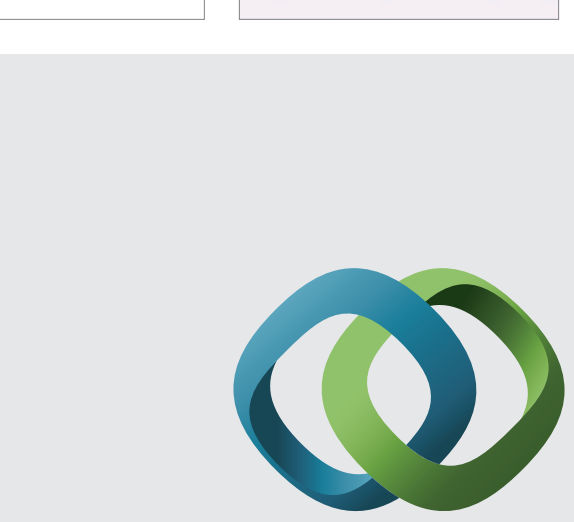

\section{Hindawi}

Submit your manuscripts at

http://www.hindawi.com
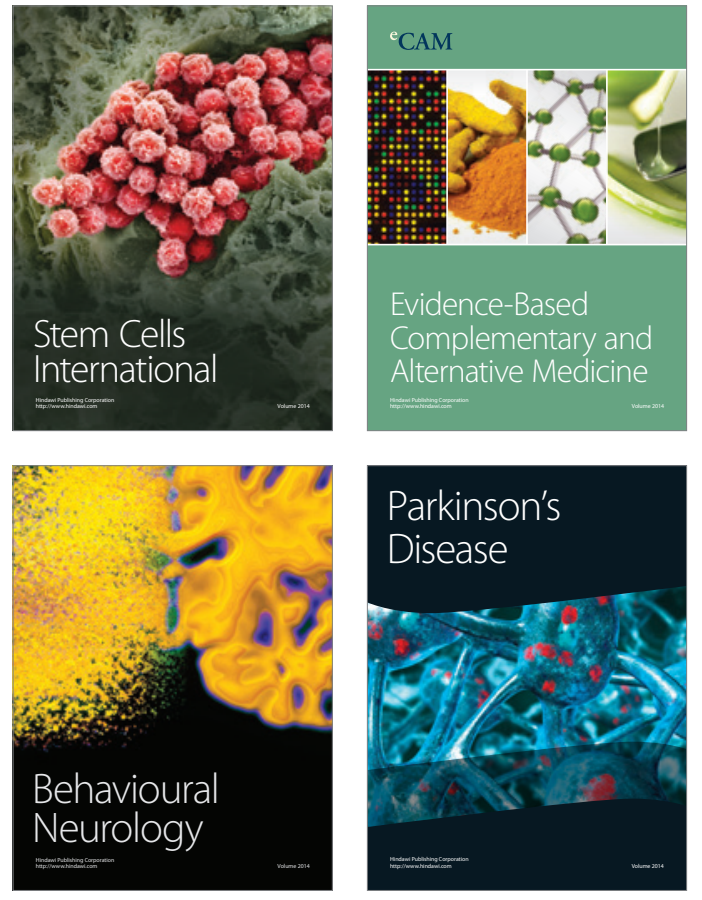
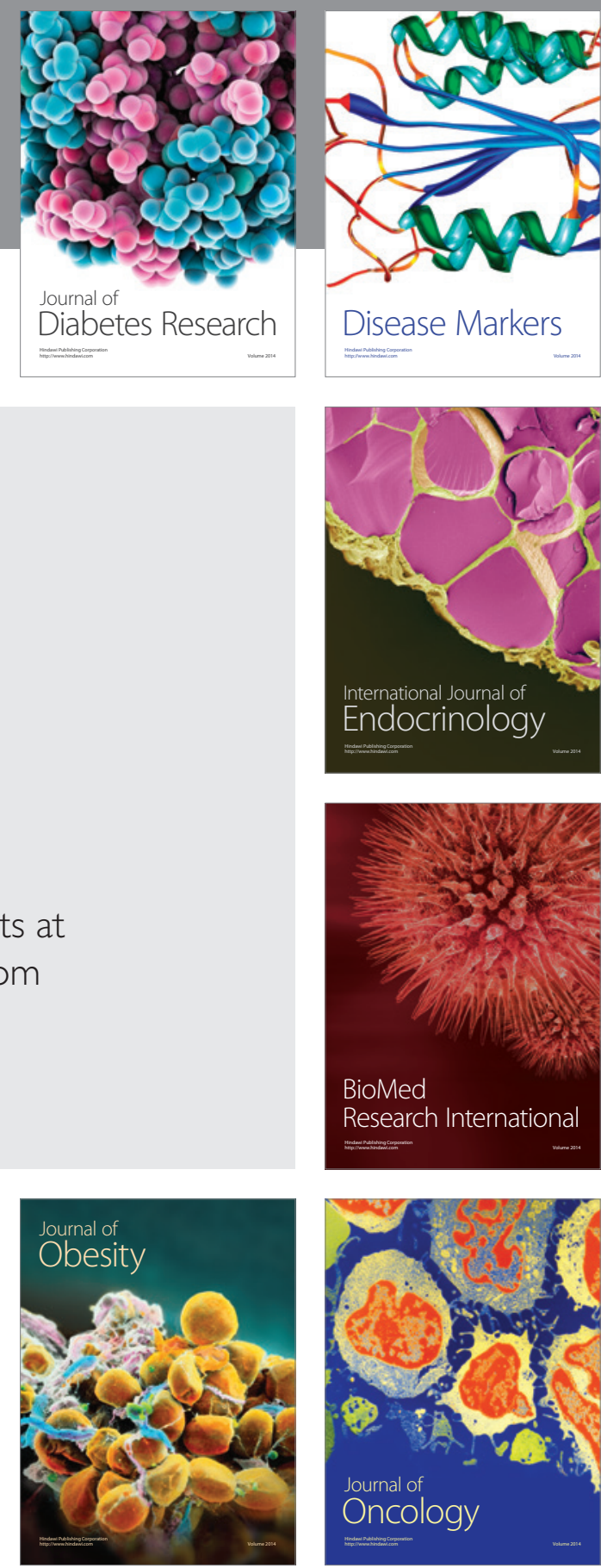

Disease Markers
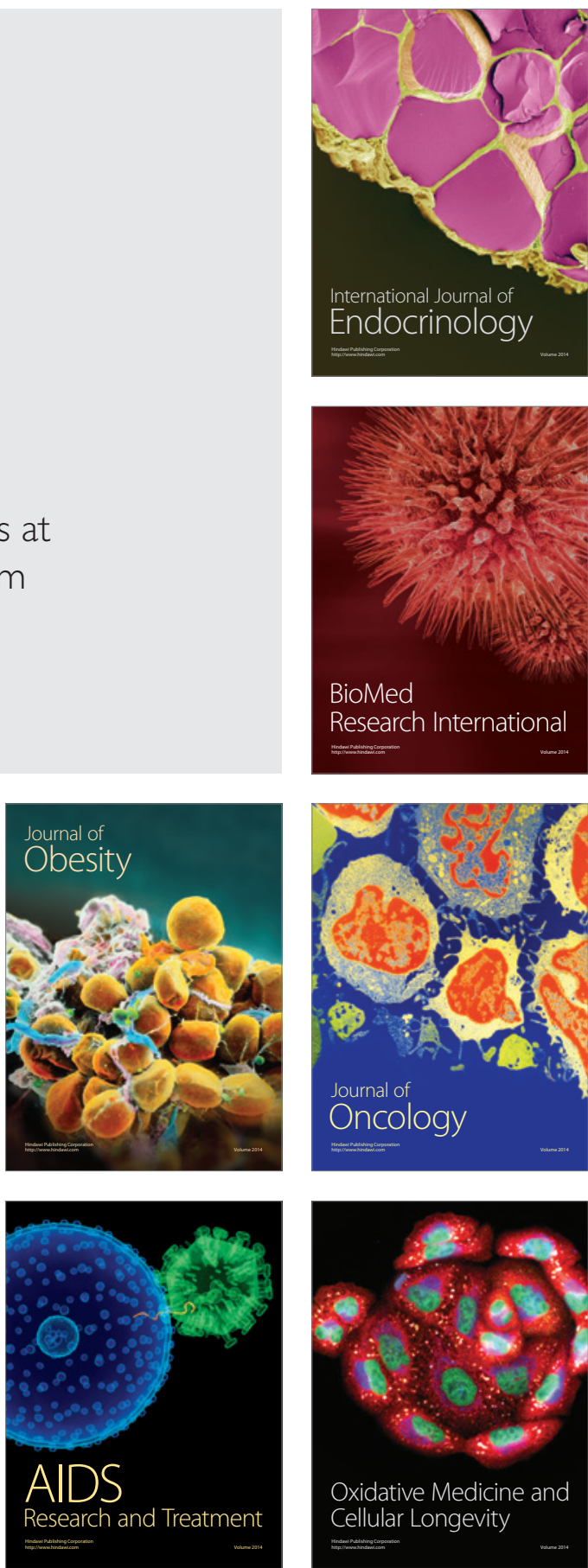